\title{
Are lampbrush chromosomes unique to meiotic cells?
}

\author{
Joseph G. Gall
}

Published online: 22 December 2012

(C) Springer Science+Business Media Dordrecht 2012

\begin{abstract}
Lampbrush chromosomes (LBCs) are transcriptionally active chromosomes found in the germinal vesicle $(\mathrm{GV})$ of large oocytes of many vertebrate and invertebrate animals and also in the giant singlecelled alga Acetabularia. These cells are all in prophase of the first meiotic division. Nevertheless, many meiotic cells do not develop LBCs, arguing that LBCs are not an essential feature of meiosis. LBCs probably represent the most active transcriptional state that can be attained by cells that must give rise to diploid progeny. Polyploidy permits cells to reach higher rates of transcription per nucleus but precludes a return to diploidy. In this sense, LBCs represent a relatively inefficient transcriptional compromise employed by large meiotic cells. These considerations help to explain why transcriptionally active GVs develop LBCs, but they do not explain why LBCs have never been seen in somatic cells, diploid or otherwise. If LBCs are truly limited to germ cells, then some of their unusual features may reflect reprogramming of the genome. If this is the case, LBCs provide unique opportunities to study reprogramming at the level of the individual transcription unit.
\end{abstract}

Responsible Editor: Herbert Macgregor.

J. G. Gall $(\bowtie)$

Department of Embryology,

Carnegie Institution for Science,

3520 San Martin Drive,

Baltimore, MD 21218, USA

e-mail: gall@ciwemb.edu
Keywords lampbrush chromosome $\cdot$ meiosis . oocyte $\cdot$ oogenesis $\cdot$ polyploidy reprogramming . transcription
Abbreviations
GV Germinal vesicle
LBC Lampbrush chromosome
Pol II Polymerase II
TU Transcription unit

\section{Introduction}

When Herbert Macgregor asked me to write an introductory essay on LBCs for this volume of Chromosome Research, my original response was to outline the major structural features of these remarkable chromosomes, discuss briefly where they occur, stress their unusually high rate of transcription, and dodge the issue of why they have been found only in meiotic cells. The focus of this essay began to shift, however, when members of my lab and I struggled to understand the results of RNAseq experiments that we carried out during the past 2 years.

In hopes of learning more about $\mathrm{LBC}$ transcription, we analyzed RNA from germinal vesicles (GVs) and oocyte cytoplasm of Xenopus tropicalis (Gardner et al. 2012). The results for the cytoplasm were not exceptional. The cytoplasm contains spliced transcripts derived from thousands of genes, as described earlier by John Gurdon's group (Simeoni et al. 2012). However, 
the GV RNA came as a complete surprise. Instead of seeing nascent transcripts from the LBCs, as we expected, we found only stable sequences derived from the introns of transcribed genes. It will take some time to understand the significance of the stable intronic sequence RNA, which persists into the embryo until at least the blastula stage, when transcription begins. But the absence of nascent transcripts in our data was easily explained, once we put our minds to the transcriptional problem faced by large germ cells. We had not fully thought through what has been known for a long time about transcription in the GV (Davidson 1986).

\section{LBCs and transcription}

A major problem for the Xenopus oocyte is to produce the transcripts needed for protein synthesis during oogenesis and early embryogenesis. The strategy used by Xenopus and many other organisms with large oocytes is to develop transcriptionally active LBCs, which produce much more RNA per chromosome than the chromosomes of small somatic cells. This is the "standard" explanation for the occurrence of LBCs in large oocytes and is correct as far as it goes.

However, it is useful to compare the LBC strategy with other ways of controlling the rate of transcription. As used here, "rate of transcription" will mean the number of transcripts or total amount of RNA derived from a gene or set of genes per unit time. The rate of elongation, which is the number of bases traversed by an RNA polymerase II (pol II) molecule per unit time, plays only a small part: the rate of elongation varies to a certain extent, but the upper limit is about $3.8 \mathrm{~kb} / \mathrm{min}$ (Ardehali and Lis 2009). Instead, the packing of pol II molecules on the template controls the rate of transcription; the more polymerases there are on a given length of DNA, the more transcripts will be produced per unit time. There is obviously a physical limit to the number of polymerases that can be packed onto a given length of DNA. The critical point for the discussion of LBCs is that oocyte transcription units (TUs) may reach the physical limit for close packing of polymerase molecules. This was shown by the elegant electron micrographs published many years ago by Oscar Miller and his colleagues (Miller and Hamkalo 1972; Hamkalo and Miller 1973), as well as by others who used his technique (Scheer et al. 1979; Hill and Macgregor 1980).

Once a TU is fully occupied by polymerase molecules, that TU has reached its maximal rate of transcription. But the GV is still only one $4 \mathrm{C}$ nucleus, and in the case of Xenopus, it must produce transcripts for a cell that will eventually reach a volume $10^{5}$ or $10^{6}$ times that of a somatic cell (Fig. 1a). To do this, it must not only transcribe at a high rate - i.e., make LBCs - but it must do two other things. It must make unusually stable transcripts, which it stores in the cytoplasm, and it must continue to do so for several months (see discussion of actual rates in Davidson 1986).
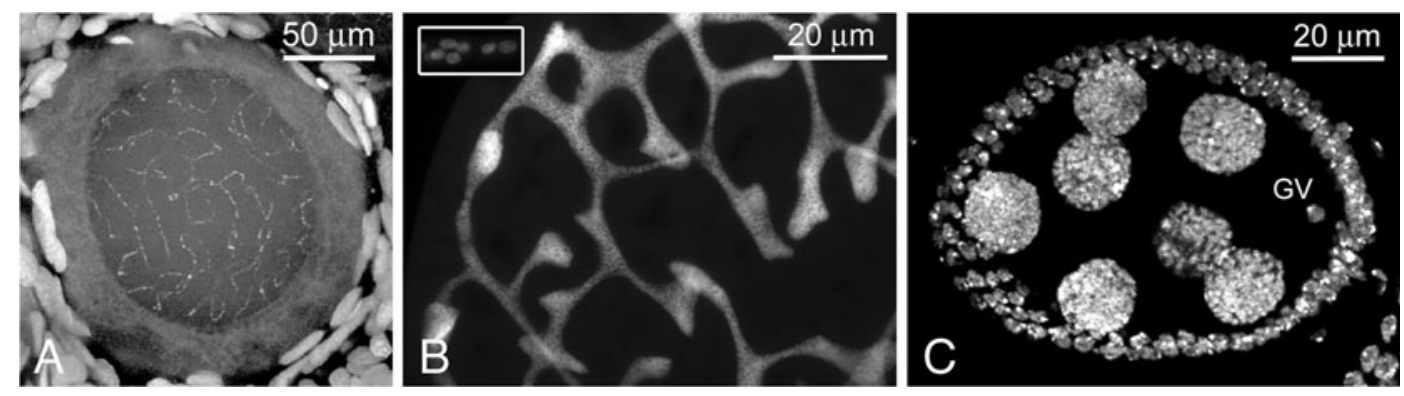

Fig. 1 Various nuclei to illustrate the enormous range in DNA content of different cell types. All stained with 4',6-diamidino-2phenylindole (DAPI), whose intensity is proportional to the amount of DNA. a and $\mathbf{c}$ are from stacks of confocal images, showing only part of the specimen. $\mathbf{b}$ is a conventional image of a relatively flat specimen. a A small oocyte from the axolotl Ambystoma mexicanum surrounded by diploid follicle cells. The axes of the LBCs are visible as DAPI-positive rows of chromomeres, but the loops are not detectable with this stain. The chromosomes contain only the 4C amount of DNA, despite the large size of the GV. b A giant lobulated nucleus from a Malpighian tubule of the caterpillar Pseudoplusia sp. The inset at the upper left shows a group of presumably diploid nuclei from a nearby field. The ploidy level of the giant nucleus is probably in the tens of thousands. c An egg chamber of Drosophila melanogaster showing the small GV with transcriptionally inactive chromatin (4C DNA content) and 7 of the 15 highly polyploid nurse cell nuclei (up to $\sim 4,000 \mathrm{C}$ DNA content). Essentially all of the transcripts of the oocyte are derived from the nurse cell nuclei. Image provided by Zehra Nizami 


\section{Polyploidy as a means to increase transcription}

Another way to increase the overall rate of transcription per nucleus is to increase the number of templates. Cells have evolved several ways of doing this, the most common being polyploidization of the entire genome or most of the genome. Polyploidization is found throughout the plant and animal kingdoms, wherever there are large terminally differentiated cells. As might be expected, the largest cells tend to have the largest nuclei with ploidy levels in extreme cases up to100,000 times the diploid value (Fig. 1b). Well-known examples of polyploidy include the macronucleus of ciliated protozoa (Blackburn and Karrer 1986), the giant lobulated nuclei in the silk gland of Bombyx (Gage 1974), Drosophila salivary gland nuclei (Swift 1962), mammalian trophoblast nuclei (Varmuza et al. 1988), and the nuclei found in the giant neurons of gastropods like Aplysia (Lasek and Dower 1971).

None of these giant somatic nuclei use LBCs as their way of increasing the rate of transcription. A reasonable explanation is that a polyploid cell can reach very high levels of transcription (per nucleus) without increasing the loading of polymerase on its genes. Seen in this light, LBCs are a relatively inefficient way for a nucleus to increase the overall rate of transcription. Those of us who study LBCs have been so impressed by their high rate of transcription per chromatid that we forget the weeks or months it takes for them to supply the oocyte with its final store of transcripts.

This brings us to a crucial question: why doesn't the oocyte simply become highly polyploid? Here, the answer is obvious or at least seemingly obvious. The oocyte must eventually complete meiosis and transmit a single set of chromosomes to the next generation. So far as is known, once a cell has become polyploid, there is no way for it to return to the diploid state. This being the case, the GV does not have the option of becoming polyploid.

The discussion so far explains why small oocytes can manage without LBCs - they can produce enough RNA with a relatively low rate of transcription. And it explains why many large oocytes do have LBCs. Because of their reproductive strategy, they have the luxury of slow development and so can manage with relatively inefficient LBCs. But what if an organism's reproductive strategy involves large oocytes that develop rapidly? Surprisingly, they sometimes become polyploid, but with a twist that is masked by the terminology we use to describe them.

\section{Polyploid oocytes}

The Drosophila oocyte provides the example (Fig. 1c). At maturity, it approaches the size of an amphibian oocyte, yet it takes only 3 days to reach full size after leaving the germarium as a relatively small cell (Spradling 1993). As is well known, the Drosophila GV itself is transcriptionally silent (or nearly so, Mahowald and Tiefert 1970). Instead, the oocyte receives transcripts from 15 highly polyploid nurse cells. The giant nurse cell nuclei are quite remarkable, undergoing 10-12 endocycles to reach DNA levels up to 1,000-4,000 times the diploid amount (Dej and Spradling 1999) (Fig. 1c). Eventually, the nurse cells dump their cytoplasm into the oocyte, and the polyploid nuclei disintegrate. The single (4C) GV then proceeds through the meiotic divisions.

An equally valid way of describing the Drosophila egg chamber is to say it is a syncytium of 16 oocytes containing $16 \mathrm{GVs}$, of which 15 become polyploid and later disintegrate. That nurse cells are modified oocytes is well known and to describe them as separate cells is at least in part a terminological issue, since they are all interconnected through the ring canals. They come from four divisions of what in other organisms might be called an oogonium, and at least one nurse cell nucleus regularly forms synaptonemal complexes before "regressing" and becoming polyploid (King 1970). So an oocyte nucleus can become polyploid, so long as it sacrifices itself for the good of one of its sisters. Not all insects use the Drosophila strategy. For instance, the grasshopper Locusta has large oocytes that develop relatively slowly over a period of weeks or months. Instead of nurse cells, its oocyte has a single large GV with LBCs (Bier et al. 1969).

\section{Do somatic nuclei have LBCs?}

This brings us to the issue that has been at the center of LBC discussions for many years - the relationship between LBCs and meiosis. LBCs have been described from dozens of different organisms, both vertebrate and invertebrate, but only from cells in meiosis (Callan 1986). This is true even for the giant single-celled alga 
Acetabularia, whose giant nucleus contains LBCs in prophase of the first meiotic division (Spring et al. 1975; Berger et al. 1994). Why have LBCs not been found elsewhere, specifically in somatic nuclei? There are two possible answers to that question. The first is that they $d o$ exist somewhere and have simply not been discovered or recognized. In the discussion above, we stressed that polyploidy and LBCs are alternative ways of increasing transcription rates in large cells. It is easy to understand why a large GV cannot become polyploid, but it is not obvious why a large somatic cell should avoid LBCs as a means of increasing its rate of transcription or why there could not be a somatic cell with a polyploid number of LBCs. Nevertheless, I am unaware of any convincing report of LBCs in a somatic cell.

\section{What is special about meiosis and LBCs?}

The other possibility is that LBCs are truly limited to germ cells. The reason cannot be that they are required for the formation of germ cells. Drosophila and many other organisms that carry out meiosis without LBCs demonstrate this fact clearly. The absence of LBCs from somatic cells suggests that we should pose the question in another way: Is there some difference between a germ cell and a somatic cell that permits the one to form LBCs while preventing the other from doing so? This question in turn focuses on the very definition of a LBC. Most of us in the LBC field have stressed that LBCs serve as a useful structural model for chromosomes in general-active transcription occurs on loops of decondensed chromatin that project from regions of inactive chromatin. We have assumed that the major difference between LBCs and somatic chromosomes is the denser packing of polymerases on the LBC loops and hence their visibility by conventional microscopy.

But suppose that LBC transcription differs in more fundamental ways from that in somatic cells. There are hints that LBCs may transcribe sequences that are not normally transcribed in other cells. A detailed analysis of the histone gene locus of the newt Notophthalmus revealed extensive read-through transcription involving much more than the coding regions themselves (Gall et al. 1983; Diaz and Gall 1985). Is this true for other loci in the oocyte, and if so, what is its significance? Other studies suggest that many repetitive sequences are transcribed on LBCs that may not be transcribed in somatic cells (Macgregor and Andrews 1977; Varley et al. 1980; Solovei et al. 1996; Deryusheva et al. 2007).

Whatever the specific differences may be between a LBC and a somatic chromosome, it is clear that the environment of the GV plays a crucial role. This is demonstrated by experiments in which sperm heads of Xenopus are injected into the GV of a living oocyte (Gall and Murphy 1998). Within minutes after injection, the chromatin of the sperm head expands dramatically and begins transcription. Eventually, each sperm head gives rise to a set of giant LBCs, comparable in every respect to the endogenous LBCs, except that they are single chromatids with unpaired loops. Even more remarkable is the ability of the amphibian GV to induce similar changes in sperm heads of heterologous species, such as the zebrafish. Most telling is the formation of LBCs from the chromatin of human sperm heads (Liu and Gall 2012). Since human chromosomes never normally form LBCs, the oocyte environment must have the ability to reprogram essentially any chromatin into the LBC state.

What specifically happens to chromatin when it is placed in the GV environment? If we could answer that question, we could probably say why LBCs are limited to germ cells. A good start is to look for features unique to oocyte chromatin. Changes in DNA methylation and histone modifications are candidates for "opening up" the genome to more extensive transcription (Morgan et al. 2012). We need not postulate transcription from all genes as a defining feature of LBCs and certainly not from the entire genome (as evidenced by the large fraction of inactive chromatin in the chromomeres). The germ cell presumably has its own specific repertoire of active genes, like any other cell. But in addition to loading more polymerases than is possible in a somatic cell, it probably transcribes sequences that are not ordinarily transcribed.

However, the simple truth is that we do not know on a global scale what sequences are transcribed by LBCs, and we will not know this until the nascent transcripts are sequenced. Our study of cytoplasmic transcripts in X. tropicalis (Gardner et al. 2012) and comparable data from Gurdon's group (Simeoni et al. 2012) tell us only what sequences are stored as spliced mRNA. As deep sequencing becomes ever more sensitive, it will be possible to examine the nascent transcripts at all loci on the LBCs and compare them with 
the nascent transcripts of somatic cells. Only then can we begin to understand what is unique about the relationship between LBCs and meiosis.

Acknowledgments I thank all members of my laboratory for constructive criticism of the ideas developed here, especially Zehra Nizami, Svetlana Deryusheva, and Jun Wei Pek. Research reported in this publication was supported by the National Institute of General Medical Sciences of the National Institutes of Health under award number R01 GM33397. The content is solely the responsibility of the author and does not necessarily represent the official views of the National Institutes of Health. JGG is American Cancer Society Professor of Developmental Genetics.

\section{References}

Ardehali MB, Lis JT (2009) Tracking rates of transcription and splicing in vivo. Nat Struct Mol Biol 16:1123-1124

Berger S, Menzel D, Traub P (1994) Chromosomal architecture in giant premeiotic nuclei of the green alga Acetabularia. Protoplasma 178:119-128

Bier K, Kunz W, Ribbert D (1969) Insect oogenesis with and without lampbrush chromosomes. Chromosomes Today 2:107-115

Blackburn EH, Karrer KM (1986) Genomic reorganization in ciliated protozoans. Annu Rev Genet 20:501-521

Callan HG (1986) Lampbrush chromosomes. Springer, Berlin

Davidson EH (1986) Gene activity in early development. Academic, Orlando

Dej KJ, Spradling AC (1999) The endocycle controls nurse cell polytene chromosome structure during Drosophila oogenesis. Development 126:293-303

Deryusheva S, Krasikova A, Kulikova T, Gaginskaya E (2007) Tandem 41-bp repeats in chicken and Japanese quail genomes: FISH mapping and transcription analysis on lampbrush chromosomes. Chromosoma 116:519-530

Diaz MO, Gall JG (1985) Giant readthrough transcription units at the histone loci on lampbrush chromosomes of the newt Notophthalmus. Chromosoma 92:243-253

Gage LP (1974) Polyploidization of the silk gland of Bombyx mori. J Mol Biol 86:97-108

Gall JG, Murphy C (1998) Assembly of lampbrush chromosomes from sperm chromatin. Mol Biol Cell 9:733-747

Gall JG, Diaz MO, Stephenson EC, Mahon KA (1983) The transcription unit of lampbrush chromosomes. In: Subtelny $\mathrm{S}$, Kafatos F (eds) Gene structure and regulation in development. Alan R. Liss, New York, pp 137-146

Gardner EJ, Nizami ZF, Talbot CC Jr, Gall JG (2012) Stable intronic sequence RNA (sisRNA), a new class of noncoding RNA from the oocyte nucleus of Xenopus tropicalis. Genes Dev 26:2550-2559
Hamkalo BA, Miller OL Jr (1973) Electronmicroscopy of genetic activity. Annu Rev Biochem 42:379-396

Hill RS, Macgregor HC (1980) The development of lampbrush chromosome-type transcription in early diplotene oocytes of Xenopus laevis: an electron microscope analysis. J Cell Sci 44:87-101

King RC (1970) Ovarian development in Drosophila melanogaster. Academic, New York

Lasek RJ, Dower WJ (1971) Aplysia californica: analysis of nuclear DNA in individual nuclei of giant neurons. Science 172:278-280

Liu JL, Gall JG (2012) Induction of human lampbrush chromosomes. Chromosome Res. doi:10.1007/s10577-012-9331-y

Macgregor HC, Andrews C (1977) The arrangement and transcription of 'middle repetitive' DNA sequences on lampbrush chromosomes of Triturus. Chromosoma 63:109-126

Mahowald AP, Tiefert M (1970) Fine structural changes in the Drosophila oocyte nucleus during a short period of RNA synthesis. Wilhelm Roux' Arch Entwicklungsmech Org 165:8-25

Miller OL Jr, Hamkalo BA (1972) Visualization of RNA synthesis on chromosomes. Int Rev Cytol 33:1-25

Morgan GT, Jones P, Bellini M (2012) Association of modified cytosines and the methylated DNA-binding protein MeCP2 with distinctive structural domains of lampbrush chromatin. Chromosome Res. doi:10.1007/s10577-0129324-x

Scheer U, Spring H, Trendelenburg MF (1979) Organization of transcriptionally active chromatin in lampbrush chromosome loops. The Cell Nucleus 7:3-47

Simeoni I, Gilchrist MJ, Garrett N, Armisen J, Gurdon JB (2012) Widespread transcription in an amphibian oocyte relates to its reprogramming activity on transplanted somatic nuclei. Stem Cells Dev 21:181-190

Solovei IV, Joffe BI, Gaginskaya ER, Macgregor HC (1996) Transcription of lampbrush chromosomes of a centromerically localized highly repeated DNA in pigeon (Columba) relates to sequence arrangement. Chromosom Res 4:588-603

Spradling AC (1993) Developmental genetics of oogenesis. In: Bate M, Martinez-Arias A (eds) The development of Drosophila melanogaster. Cold Spring Harbor Laboratory Press, Cold Spring Harbor, pp 1-70

Spring H, Scheer U, Franke WW, Trendelenburg MF (1975) Lampbrush-type chromosomes in the primary nucleus of the green alga Acetabularia mediterranea. Chromosoma 50:25-43

Swift H (1962) Nucleic acids and cell morphology in dipteran salivary glands. In: Allen JM (ed) The molecular control of cellular activity. McGraw Hill, New York, pp 73-125

Varley JM, Macgregor HC, Erba HP (1980) Satellite DNA is transcribed on lampbrush chromosomes. Nat (Lond) 283:686-688

Varmuza S, Prideaux V, Kothary R, Rossant J (1988) Polytene chromosomes in mouse trophoblast giant cells. Development 102:127-134 